Investigaciones Turísticas

ISSN: 2174-5609

\title{
El Turismo Residencial en Valle de Bravo, México. Una Interpretación de su Ciclo de Vida
}

\author{
Marie Eugenie Deverdun Reyna \\ Universidad Autónoma del Estado de México \\ México \\ geniedev80@gmail.com \\ Maribel Osorio García \\ Universidad Autónoma del Estado de México \\ México \\ maribelosorio2@gmail.com \\ Alfonso Iracheta Cenecorta \\ Colegio Mexiquense A.C. \\ México \\ axic@cmq.edu.mx
}

\section{RESUMEN}

El turismo residencial en Valle de Bravo (Estado de México) surge a partir de la consolidación de su presa, iniciando un proceso de transformación no sólo paisajística, sino territorial y socioeconómica, el cual además, ha marcado la pauta para llevar a este destino a convertirse en uno de los sitios turísticos más importantes del Estado de México. El presente artículo tiene como objetivo realizar una caracterización territorial y urbana de las etapas que ha tenido Valle de Bravo debido a la proliferación de residencias desde sus inicios como sitio turístico hasta la época actual, analizando su posible tendencia de crecimiento para los próximos años. Para poder realizar este análisis, se utilizó el modelo del Ciclo de Vida Turístico en el que diversas variables cuantitativas y cualitativas fueron utilizadas para ejemplificar cómo ha ido evolucionando Valle de Bravo. A través de éste análisis se demuestra que la zona está acercándose a límites de capacidad de carga, lo cual supone un mayor impacto en la zona principalmente en sus recursos más importantes: el espacio físico y, su alto valor paisajístico y natural.

Palabras clave: Turismo residencial, ciclo de vida, Valle de Bravo 


\title{
Investigaciones Turísticas
}

ISSN: 2174-5609

Investigaciones

TURISTICAS

\section{Residential Tourism in Valle de Bravo, Mexico. An Interpretation of its Life Cycle}

\author{
Marie Eugenie Deverdun Reyna \\ Universidad Autónoma del Estado de México \\ México \\ geniedev80@gmail.com \\ Maribel Osorio García \\ Universidad Autónoma del Estado de México \\ México \\ maribelosorio2@gmail.com \\ Alfonso Iracheta Cenecorta \\ Colegio Mexiquense A.C. \\ México \\ axic@cmq.edu.mx
}

\begin{abstract}
Second home tourism in Valle de Bravo (Mexico State) arose from the consolidation of its dam, which initiated a transformation process not only in terms of its landscape, but also on a territorial and socio-economic level, which paved the way for this destination to become one of the most important tourist areas of the State of Mexico. The objective of this paper is to carry out a characterisation of the stages that Valle de Bravo has undergone, due to the proliferation of residences since its beginnings as a tourist destination until the present day, analysing its possible growth trend for the next few years. In order to perform this analysis, we have used the tourism life cycle model, in which various quantitative and qualitative variables have been used to exemplify how Valle de Bravo has evolved. This analysis shows that the area is approaching its load capacity limit, which means that the area will suffer a greater impact, mainly in terms of its most important resources: the physical space and its high landscape and natural value.
\end{abstract}

Keywords: Second home tourism, lifecycle, Valle de Bravo 


\section{INTRODUCCIÓN}

En las últimas décadas el turismo se ha diversificado conformando una serie de nuevas tendencias y modalidades que ha acaparado diversos estudios e investigaciones en todo el mundo dentro de las cuales el Turismo Residencial (TR) no ha sido la excepción. Si bien no es un término nuevo, esta expresión se incluyó en el debate académico en 1979 por el autor Francisco Jurdao y a partir de esa fecha ha sido utilizada por diferentes investigadores para hacer referencia a un variado conjunto de procesos difíciles de delimitar, cuyo contexto más significativo lo forman las regiones turísticas de las riberas, entremezclándose con una intensa actividad económica que gira en torno al negocio inmobiliario con aspectos propios del turismo tradicional y éstos, a su vez, con algunas de las nuevas formas migratorias y de residencialidad.

Fue a partir de 1970 que en España el turismo residencial acaparó la atención de diversos investigadores al observar los cambios sociales que se daban en diversas zonas a causa de la venta de suelo agrícola, propiedad de campesinos, a empresarios urbanizadores. Tras el cambio de propiedad, el suelo era destinado a la construcción de urbanizaciones turísticas de chalets que eran adquiridos principalmente por jubilados del norte de Europa. Estas observaciones derivaron en los primeros estudios que existen de este tipo de modalidad turística, la cual ha sido, hasta la fecha, parte de diversos debates académicos que van desde sus diversas perspectivas de estudio, hasta la propuesta de no considerarlo turismo residencial, sino una práctica inmobiliaria que deriva en todo tipo de impactos.

De los temas más estudiados sobre el turismo residencial, se destaca el de la migración internacional (Benson y O’Reilly, 2009; Hall y Müller, 2004; Müller, 2002; Rodríguez, 2004; Williams y Hall, 2000), ya que en un inicio, el turismo residencial comenzó como un fenómeno migratorio que se dio desde el norte al sur de Europa (Alarcón et al., 2010; Casado et al., 2004; Huete y Mantecón, 2010 ; López, 1995; O’Really, y Benson, 2009), se mencionan también los estudios del perfil que tienen los turistas residenciales (Coles et al., 2005; O’Really y Benson, 2009; Sanz, 2008), y los de medición estadística (Varela et al., 2003), así como su análisis teórico (Guilding et al., 2005).

La producción científica española sobre esta modalidad turística, es una de las más vastas, pues además de los temas mencionados anteriormente se han estudiado los conflictos territoriales por la apropiación y saturación del espacio (Almeida y López, 2003; Díaz, 2004; Díaz y Lourés, 2006; Fernández y Barrado, 2011; García et al., 2002; Gili, 2003; Mazón, 2006 ; Varela et al., 2003), así como impactos territoriales y sectoriales provocados por el turismo residencial (Aledo, 2008 ; Aledo et al., 2010; Torres, 2003). Los estudios sobre comunidades receptoras de turismo residencial (Huete y Mantecón, 2010; Mantecón y Huete, 2011; Mazón et al., 2009); los relacionados con cuestionamientos teórico - conceptuales (Aledo et al., 2007; Huete et al., 2008). Y las investigaciones sobre sus posibles tendencias de desarrollo (Huete, et al., 2008; Salvá, 2011). 
En México, el tema se comienza a estudiar a raíz de la presencia de segundas residencias en zonas costeras, principalmente Acapulco y la zona norte del país, observando este fenómeno se realizan diversos estudios para comprender las dinámicas del turismo residencial, notando su proliferación no sólo en zonas de litorales o fronterizas, sino al interior del país destacando la construcción de este tipo de viviendas en los estados de México y Morelos principalmente. En el año 2004 surge uno de los estudios más importantes relacionados con el Turismo de Segundas Residencias en México, y es el realizado por la Universidad Autónoma Metropolitana en conjunto con el Centro de Estudios Superiores de Turismo de la Universidad Autónoma del Estado de México; para desarrollar este estudio se seleccionaron 15 destinos dentro de la República Mexicana, los cuales marcan claras características relacionadas con el turismo residencial, tal es el caso de: Acapulco, Cancún, Tijuana, Cuernavaca, Tepoztlán, Valle de Bravo, entre otros. El objetivo que tuvo esta investigación fue el de conocer las características y el valor de mercado que genera el turismo nacional e internacional en segundas residencias; así como de los retos y oportunidades que representa este turismo para los destinos que cuentan con esta infraestructura (UAM/CESTUR, 2004). Dicho estudio estuvo dirigido por el Dr. Daniel Hiernaux - Nicolás.

Dentro de esta investigación, se observa que Valle de Bravo en el Estado de México, es uno de los sitios en los que detona el turismo residencial entre los 70's y 80's, provocando una profunda transformación territorial. Es indudable que esta zona ha logrado su crecimiento y consolidación gracias al turismo y a la construcción de segundas residencias, por lo que este artículo tiene como objetivo realizar una caracterización urbana y territorial de las etapas que ha tenido Valle de Bravo debido a la proliferación de residencias desde sus inicios como sitio turístico en 1950, hasta la época actual, identificando la etapa en la que se encuentra en este momento, así como su posible trayectoria para los próximos años.

El modelo teórico utilizado para este caso, es el modelo del ciclo de vida turístico propuesto por Butler, el cual ha sido utilizado como referencia para describir la evolución que tienen los destinos turísticos, siendo además, una de las aportaciones más debatidas en relación a esta cuestión. El modelo propone el análisis del comportamiento de la demanda y de la capacidad de carga de los centros turísticos y prevé una relación positiva entre el incremento del número de visitantes y el desarrollo turístico; además incluye el tema del desarrollo del área, representando un modelo complejo y flexible por la cantidad de variables que pueden integrarse permitiendo centrarse en el análisis de una, algunas, todas las variables o su interrelación, brindando la oportunidad de obtener interesantes resultados ya que analiza el destino como un sistema en donde cada una de sus partes es un eslabón que refleja la realidad del destino (Vera et al., 1997).

Para explicar este proceso el trabajo se articula de la siguiente forma: en principio se detalla el marco referencial de la zona de estudio; en un segundo y tercer apartado se 
incluye el fundamento teórico - metodológico; en la cuarta parte se realiza la aplicación del Modelo de Ciclo de Vida Turístico (CVT); en la quinta parte se detalla la situación actual con relación al crecimiento poblacional, de vivienda y de requerimiento de suelo; finalmente se aportan las conclusiones de la investigación.

\section{MARCO REFERENCIAL SOBRE VALLE DE BRAVO}

El municipio de Valle de Bravo se ubica en el poniente del Estado de México, a 96 $\mathrm{km}$ de la ciudad de Toluca y a $145 \mathrm{~km}$ de la Ciudad de México. Colinda al norte con el municipio de Donato Guerra, al oriente con Amanalco de Becerra, al sur con Temascaltepec, y al poniente con Ixtapan del Oro, Santo Tomás de los Plátanos y Otzoloapan. La superficie del municipio es de $421.95 \mathrm{~km}^{2}$, la cual representa el $1.87 \%$ del territorio estatal (PMDU, 2006).

\section{Mapa 1: Localización geográfica de Valle de Bravo, México}

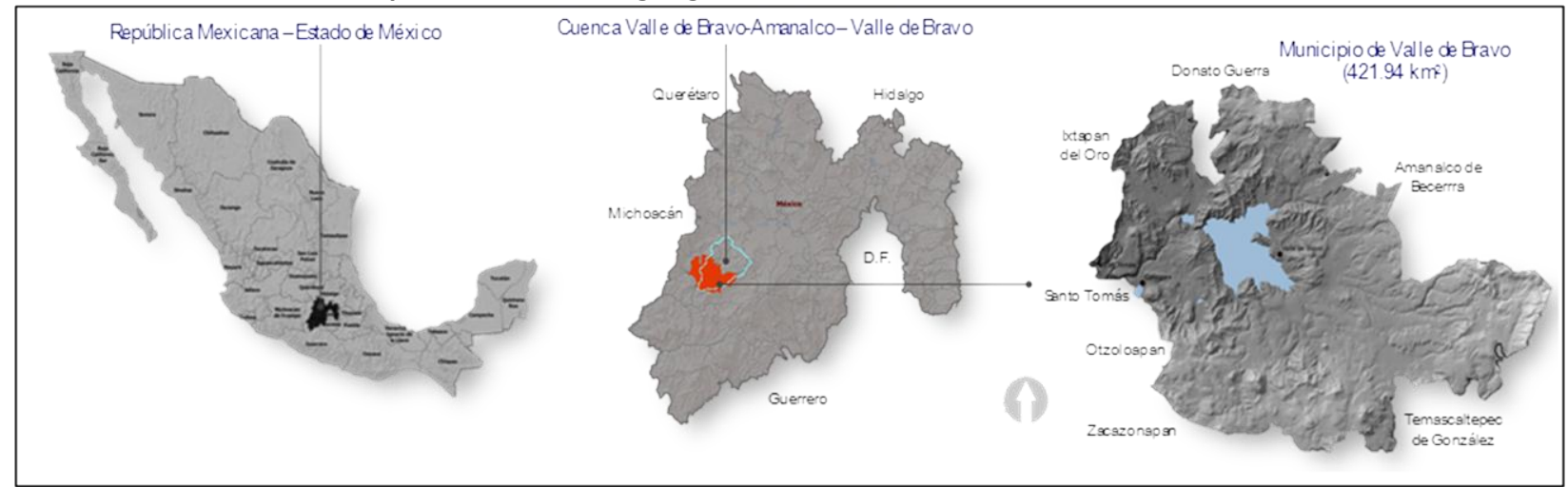

Fuente: Ilustración tomada de (Iracheta et al., 2014)

El clima en el municipio es templado subhúmedo, registra una temperatura promedio anual de $20^{\circ} \mathrm{C}$, con un rango máximo de $32^{\circ} \mathrm{C}$ y mínimo de $1.3^{\circ} \mathrm{C}$. En el municipio existen tres presas, la más importante es la de Valle de Bravo, tiene una extensión de $21 \mathrm{~km} 2$, actualmente pertenece a la Comisión de Aguas del Valle de México y se utiliza para abastecer de agua potable a la ciudad de Toluca y a la Zona Metropolitana de la Ciudad de México (PMDVB, 2013). Valle de Bravo está constituido por 43 localidades y 9 Áreas Naturales Protegidas (ANP). El municipio cuenta con 42,195 hectáreas, de las cuales actualmente el: $59.6 \%$ es de uso forestal, el $23.4 \%$ son áreas agropecuarias, el $7.4 \%$ son pastizales, el $4.4 \%$ son cuerpos de agua y, el 5.2\% es de uso urbano (PMDVB, 2013). 
Mapa 1: Usos de suelo. Valle de Bravo, México 2010

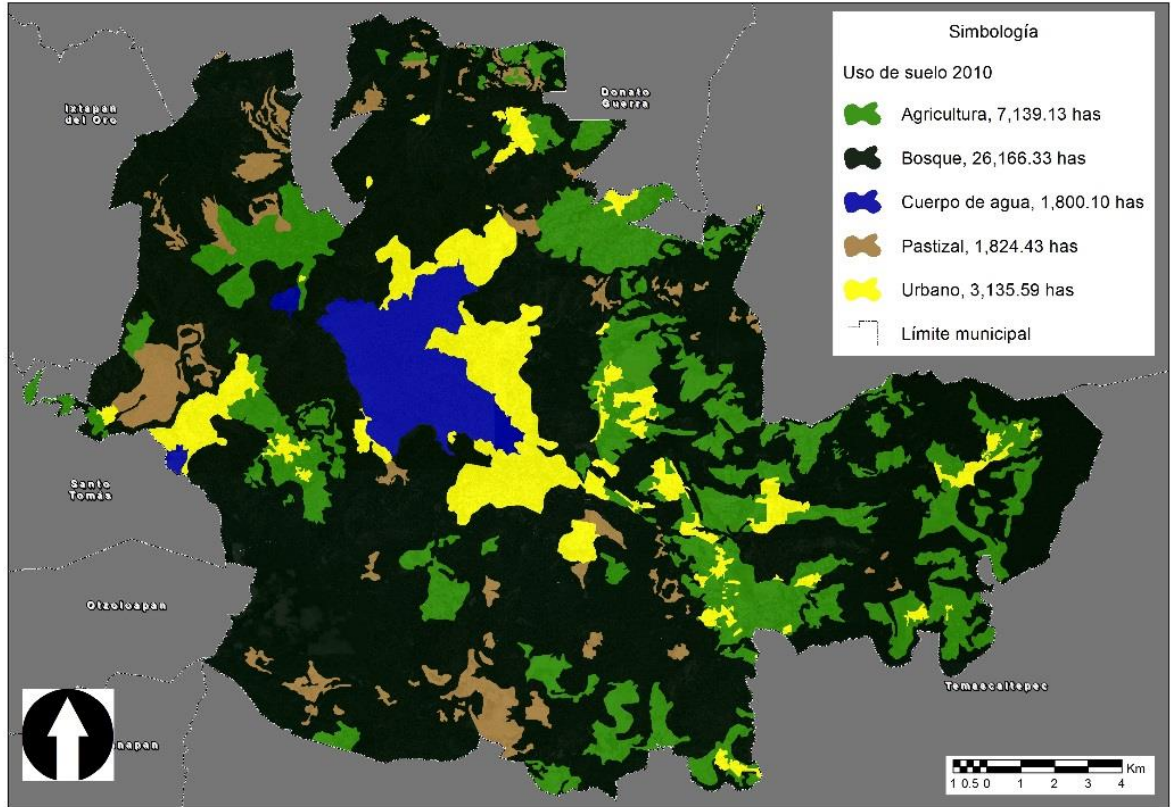

Fuente: Centro EURE. 2013.

La población de Valle de Bravo, para el 2010 alcanzó la cifra de 61.599 habitantes, con una tasa de crecimiento media anual de $2.7 \%$. El total de hogares contabilizados eran 14.778. La mayor concentración de viviendas se da en la Cabecera Municipal, ahí se localizan el $47 \%$ del total de viviendas particulares. De acuerdo con cifras de la Secretaría de Turismo, en los últimos años se ha producido un importante crecimiento en Valle de Bravo, tanto en la afluencia de visitantes (3.751.822 personas anualmente) como en derrama económica (2.617 millones de pesos anuales), situándose con ello como el sector clave del desarrollo de la economía municipal así como de generador de empleos a través de los servicios turísticos, ya que representan más del $60 \%$ de la actividad económica (SECTUR, 2013).

Dentro de esta afluencia de visitantes, se destaca la presencia de un gran número de población flotante, la cual para el 2013, se estimó que alcanzó la cifra de 27.089 personas (esto originado por el incremento de viviendas); agregando los 105.000 turistas anuales que tiene la zona, es decir 2.000 semanales, se estima que en un fin de semana común en Valle de Bravo están habitando más de 90.000 personas (Iracheta et al., 2014).

En 1971 se expidió la Ley sobre Protección y Conservación de la Ciudad de Valle de Bravo y en el 2002, se expidió el Reglamento de Imagen Urbana de Valle de Bravo, que perduró hasta el 17 de agosto de 2006, fecha en que se aprobó el nuevo Reglamento de Imagen Urbana del Municipio de Valle de Bravo (aprobado junto con el Plan de Desarrollo Urbano). Este Reglamento establece la normatividad que debe seguirse para la protección 
de la tradición arquitectónica del municipio (PMDVB, 2013). Desde 1940 Valle de Bravo es considerada zona turística; para 1971 fue declarada Ciudad Típica y en el 2005 recibe el título de "Pueblo Mágico", con el cual se consolida aún más el municipio como zona turística y cultural del Estado de México. Desde 2003 se realiza el ya tradicional Festival de las Almas, posicionándose actualmente como uno de los festivales culturales más importantes a nivel estatal.

\section{FUNDAMENTACIÓN TEÓRICA}

\subsection{El turismo residencial}

A lo largo del tiempo, diversos autores han otorgado su propia definición acerca del turismo residencial, enfocando estos conceptos de acuerdo a la visión de los propios estudios que realizan. Para este artículo se utilizará como referencia la definición creada por Tomás Mazón y Antonio Aledo: «el turismo residencial es la actividad económica que se dedica a la urbanización, construcción y venta de viviendas que conforman el sector extra-hotelero, cuyos usuarios las utilizan como alojamiento para veranear o residir, de forma permanente o semipermanente, fuera de sus lugares de residencia habitual, y que responden a nuevas fórmulas de movilidad y residencialidad de las sociedades avanzadas» (Mazón, T. et al., 2005).

Asociando el turismo residencial a la expansión de la urbanización, podemos entenderlo desde una doble perspectiva: primero el crecimiento de un espacio urbanizado; segundo, la expansión de una forma de vida, como parte de un cambio en el proceso cultural. Entendido bajo esta dinámica podemos comprender que el turismo residencial no sólo tiene un efecto en el sentido de la edificación de las casas, sino su espectro es más amplio al abarcar otros sectores y provocando toda clase de impactos (Aledo, 2002).

Para fines de esta investigación, se entenderá el turismo residencial desde la dinámica de crecimiento de un espacio urbanizado, por lo que uno de los factores que puede motivar el estancamiento del producto sería la pérdida de calidad ambiental y el agotamiento del principal recurso de este tipo de turismo, el suelo urbanizable. A este proceso Aledo (2002) lo llama fagotización del espacio. "para comprender la magnitud de los impactos provocados por la expansión urbana, hay que tener presente que, mientras en el mundo natural los flujos son mayoritariamente de carácter vertical, la urbanización es horizontal; así si se cortan, o impiden los flujos verticales de los ciclos ecológicos (atmósfera - subsuelo), resultan los terribles efectos de la expansión urbana" (Aledo, 2002). De ahí también se puede resaltar que el turismo residencial es extensivo, ya que consume una gran cantidad de espacio, provocando con esto más impactos negativos en el ambiente. 
A través de estos conceptos se observa como la transformación territorial bajo esta dinámica de turismo residencial, ha ocasionado que los destinos se modifiquen alterando los municipios; por esto que resulta pertinente analizar el crecimiento y desarrollo que tienen las zonas donde se da este tipo de turismo para realizar adecuadas planificaciones y crear sitios urbanos aptos para la vida cotidiana de sus ciudadanos y de sus visitantes.

\subsection{El Modelo del Ciclo de Vida Turístico (CVT)}

La utilización de este concepto es bastante reciente en el sector turístico y se ha aplicado especialmente en los destinos turísticos (Serra, 2011). El modelo propuesto por Butler en 1980 representa uno de los paradigmas esenciales en torno al desarrollo de los destinos turísticos (Lundtorp y Wanhill, 2001) y es uno de los enfoques de mayor influencia y más ampliamente referenciado en el análisis del desarrollo del turismo local (Harrison, 1995). Previamente autores como Gilbert (1939) y Christaller (1964) identificaron tres estadios en el progreso de los destinos turísticos en los que se detectaban ciertas regularidades en su crecimiento. Noronha (1976), Miossec (1977) o Stanfield (1978) basaron también sus aportaciones en la idea fundamental de que los destinos transcurren por una serie de fases o ciclos a lo largo de su vida (García et al., 2013).

El modelo CVT de Butler se fundamenta en la Teoría del Ciclo de Vida del Producto propuesta por Dean en el ámbito de gestión del Marketing (1950), adaptándola a los destinos turísticos como si se tratara de un producto, y teniendo en cuenta dimensiones relacionadas con las infraestructuras, actitudes de los turistas y residentes, implicación de los agentes locales y externos en la comercialización del producto, accesibilidad y competencia de los destinos (García et al., 2013).

Para Butler, los destinos inician de manera lenta para experimentar con el tiempo un crecimiento más rápido, una estabilidad y finalmente una declinación. Esta saturación se expresa en términos ambientales, (por ejemplo escasez de tierra, calidad del agua y del aire); de planta física (transportación, alojamiento, otros servicios), o de factores sociales (acelerada expansión demográfica, resentimientos en la población local, etc.) (Vera et al., 1997).

En el Cuadro 1 se muestran algunas de las características de las etapas de este modelo evolutivo: 
Cuadro 1: Etapas del Ciclo de Vida Turístico (CVT)

\begin{tabular}{|c|c|}
\hline Etapa & Características \\
\hline Exploración & $\begin{array}{l}\text { Número pequeño de turistas que realizan sus propios itinerarios. El uso de los } \\
\text { servicios locales y el contacto con residentes son constantes. Vías de acceso } \\
\text { precarias o escasas, la zona permanece alejada de las masas. }\end{array}$ \\
\hline Participación & $\begin{array}{l}\text { El turismo actúa como catalizador para las iniciativas locales. Aumenta el número de } \\
\text { visitantes. Los residentes comienzan a proveer servicios a los visitantes. Se presiona } \\
\text { a las administraciones para proveer de infraestructura para la actividad turística. Se } \\
\text { comienza a modificar el uso de suelo agrícola por comercial o turístico. }\end{array}$ \\
\hline Desarrollo & $\begin{array}{l}\text { Ya existe un área de mercado turístico bien definida. La participación de la } \\
\text { comunidad local y el control del desarrollo declinarán apresuradamente. Algunos } \\
\text { servicios locales desaparecen y surgen servicios más diseñados y actualizados por } \\
\text { organizaciones ajenas a la comunidad. Cambios en la apariencia física, los atractivos } \\
\text { naturales y culturales. Se hace necesaria la planificación de la zona para evitar los } \\
\text { impactos ya que está marcada por la especulación del suelo y la comercialización } \\
\text { intensa. }\end{array}$ \\
\hline Consolid & $\begin{array}{l}\text { El destino se encuentra completamente inmerso en una industria turística. El } \\
\text { turismo es el sector económico principal para gran parte del área. Los habitantes } \\
\text { locales comienzan a cambiar (o abandonar) sus actividades y se centran en } \\
\text { desarrollar las propias del turismo y el comercio, creando una dependencia casi } \\
\text { absoluta del sector terciario. }\end{array}$ \\
\hline Estancamiento & $\begin{array}{l}\text { El máximo número de visitantes es alcanzado y los niveles de capacidad de carga se } \\
\text { encuentran en su máximo, o son rebasados. El destino comienza a dejar de estar de } \\
\text { moda llevando a problemas sociales, ambientales y económicos en la comunidad } \\
\text { local. En esta misma etapa se generan nuevos desarrollos en las áreas periféricas del } \\
\text { destino turístico. }\end{array}$ \\
\hline $\begin{array}{c}\text { Decadencia / } \\
\text { Rejuvenecimiento }\end{array}$ & $\begin{array}{l}\text { Se consideran tres posibilidades: } 1 \text {. Declive total: Los visitantes se marchan a nuevos } \\
\text { lugares para comenzar de nuevo el ciclo. } 2 \text {. Cambio de función: La zona se puede } \\
\text { convertir en una ciudad dormitorio, o exclusiva de segundas residencias. } 3 . \\
\text { Rejuvenecimiento o recuperación: La zona turística puede elegir buscar entre nuevos } \\
\text { tipos de clientes o nuevos usos turísticos. El destino requerirá un cambio en los } \\
\text { productos turísticos ofertados así como la participación del gobierno y compañías } \\
\text { privadas. Se buscará reiniciar de nueva cuenta el ciclo de vida. }\end{array}$ \\
\hline
\end{tabular}

Fuente: Elaboración propia a partir de Aledo, 2002

De acuerdo con Aledo (2002), las aplicaciones del modelo CVT se pueden agrupar en tres áreas:

- Modelo explicativo. El CVT puede convertirse en un modelo teórico explicativo del desarrollo turístico de un resort determinado. La teoría de CVT simplemente reconoce que las áreas turísticas son dinámicas y proporciona una estructura analítica que facilita la comprensión de la evolución del resort, su introducción dentro del mercado turístico y cómo evoluciona en términos cualitativos y cuantitativos.

- Marketing y planificación. Basándonos en la comprensión que el modelo CVT otorga sobre la evolución y el desarrollo de los destinos turísticos, esta podría servir para planificar y establecer bases sostenibles de desarrollo turístico una vez que se han analizado las diferentes variables que éste modelo utiliza. En 
cada fase existe una respuesta diferente por parte de los actores involucrados, esta asociación entre fases y comportamientos permite la planificación del resort para introducir modificaciones pertinentes y evitar la "muerte" del destino.

- Predicción. Es quizá aquí donde más críticas ha recibido este modelo. El modelo CVT no debería presuponer la inevitabilidad y unilinealidad del proceso que conlleva. Es necesario incluir los cambiantes factores locales para poder interpretar y aplicar la teoría de una forma más ideográfica, y es aquí donde las transformaciones socio-espaciales pueden jugar un papel muy interesante.

Siguiendo con Aledo (2002), el éxito de este modelo no reside tanto en su capacidad de predicción como en su poder explicativo del proceso de desarrollo de un área turística. De acuerdo con Bianchi (1994), el CVT proporciona una ayuda aceptable y adecuada para la comprensión de la evolución de un destino turístico y, por lo tanto, debería servir como guía a la hora de diseñar estrategias y decisiones para la adopción y puesta en marcha de planes que pretendan establecer bases sostenibles de desarrollo para las comunidades (Aledo, 2002).

Con relación a los conceptos propuestos por el modelo CVT, Aledo (2002) realiza una aplicación de éste con el turismo residencial. El autor señala que el modelo CVT intenta explicar la evolución de un resort turístico y, para el caso del turismo residencial, la identificación del resort estaría circunscrita al territorio municipal, es decir, cada municipio será considerado un resort, porque su recurso es el espacio urbanizable, sobre los que los municipios tienen autonomía para su delimitación y ordenación.

\section{METODOLOGÍA}

Para poder realizar este análisis de transformación espacial, se utilizó el Ciclo de Vida Turístico bajo el esquema de modelo explicativo y de predicción, para lo cual diversas variables cuantitativas y cualitativas fueron usadas para ejemplificar cómo ha ido evolucionando la zona a través de las diferentes fases que el mismo modelo propone: Las variables que se utilizaron fueron: población total, población flotante, viviendas totales, segundas residencias y usos de suelo. Información obtenida del Instituto Nacional de Estadística y Geografía (INEGI), de la Secretaría de Turismo del Estado de México, y del Centro de Estudios Territoriales y Políticas Públicas (Centro Eure). También se consultaron los Planes Municipales y de Desarrollo Urbano de Valle de Bravo. Para la predicción de crecimiento de la zona y su posible tendencia, se utilizaron datos oficiales del Consejo Nacional de Población (CONAPO) en términos de proyección de crecimiento de poblacional. 
Para realizar el cálculo de la tasa de crecimiento media anual (TCMA) tanto poblacional como de vivienda, se utilizó la fórmula que propone INEGI:

$$
\begin{array}{ll} 
& \text { Donde: } \\
& \text { TCMA }=\text { Tasa de crecimiento media anual } \\
\text { TCMA }=(\text { PFinal } / \text { PInicial })^{1 / n-1}-1 & \text { PFinal = Población Final PInicial = Población Inicial } \\
\mathrm{n}=\text { Años del periodo }
\end{array}
$$

Para calcular la población flotante existen diversas metodologías: Mendizábal (1996) sistematizó un cálculo para Barcelona, el cual incluye más de 7 datos estadísticos y censales; por su parte el Instituto de Estadística de Cataluña creo la Población ETCA (Población equivalente a tiempo completo año); finalmente la Ley de Aguas 1999 de la Generalitat de Cataluña, la estima a través de la suma de la población residente más un $40 \%$ de la población estacional máxima. Este ajuste se estableció de acuerdo a lo observado en la región, y con esto estiman la población flotante de la zona (Módenes, 2006).

Estas metodologías utilizan datos locales disponibles para obtener de manera más precisa el dato de la población flotante. Por esto el presente artículo utiliza el cálculo establecido por el Plan Municipal de Desarrollo Urbano (2006), ya que esta fórmula utiliza los datos censales y estadísticos disponibles que tiene Valle de Bravo:

$$
P F=(S R)(P H V T)
$$

Dónde: $\mathrm{PF}=$ Población Flotante

$\mathrm{SR}=$ Segundas Residencias

PHVT = Promedio de Habitantes por Vivienda

La información anterior se complementó con referencias históricas acerca de la transformación de Valle de Bravo provocada por el TR, la cual se obtuvo de cinco entrevistas abiertas aplicadas a personas clave de la localidad, tal es el caso de dos ex directores de desarrollo urbano municipal, un geógrafo representante de la Comisión ProCuenca Amanalco - Valle de Bravo, el cronista municipal y una reconocida escritora. Con estas aportaciones se lograron construir las primeras etapas del ciclo de vida, así como algunos datos y proyecciones que reforzaron a las etapas más avanzadas.

\section{APLICACIÓN DEL CVT AL TURISMO RESIDENCIAL DE VALLE DE BRAVO}

A continuación se presenta la caracterización realizada en Valle de Bravo con relación a su ciclo de vida. 
Exploración. En 1938 se inician los trabajos para la construcción de la presa Valle de Bravo, la cual formaba parte del Sistema Hidroeléctrico "Miguel Alemán". Para ello se llevó a cabo la expropiación de alrededor de 3.000 hectáreas cercanas a la población denominada "El Plan". Después de la inundación de la zona, cierto grupo de inversionistas, apoyados por el gobierno federal, proyectaron el Fraccionamiento Campestre denominado "Bosques del Lago" (hoy "Avándaro"), constituyendo las empresas: Propulsora de Valle de Bravo S.A. y Avándaro S.A., pioneras en el ramo de los desarrollos urbanos y en la construcción de casas de fin de semana. Las obras del fraccionamiento fueron concluidas entre 1952 y 1954, el cual contaba con lotes de gran extensión, desde $2.400 \mathrm{~m}^{2}$ en algunas zonas, y de hasta 10.000 a $12.000 \mathrm{~m}^{2}$ en otras.

Participación. Hacia fines de 1950 se inició con el diseño del campo de golf y el proyecto del "Club de Golf Avándaro". En 1954, Gobbels realizó un interesante trabajo de investigación donde registró la difícil situación económica del pueblo como resultado de la inundación de la zona, y reportó para ese entonces, la existencia de 60 casas de fin de semana edificadas y en proceso de construcción (Gobbels, 1954). A principios de 1960 se pone en funcionamiento el citado campo de golf mediante la venta de los primeros lotes y la construcción de casas. También se construye la carretera Villa Victoria - Valle de Bravo, elemento que sería determinante en la proyección del desarrollo en las siguientes décadas al ser una vía de acceso más rápida y cómoda.

Desarrollo. Tras haber sido Valle de Bravo un lugar prácticamente desconocido durante dos décadas, debido a la celebración del festival "Rock y Ruedas" en Avándaro en 1971 (se estimó que contó con la presencia de entre 250,000 y 500,000 jóvenes) (Del Olmo y Castañeda, 2012 ), la ciudad trasciende sus fronteras y comienza a ser un sitio de interés intensamente frecuentado. En ese mismo año, Valle de Bravo fue declarada Ciudad Típica, surgiendo con esto la "Ley de Protección y Conservación de la Ciudad".

Se inicia el fenómeno de desarrollo inmobiliario en el rubro de la casa de campo, marcando una tendencia a lo que posteriormente sería la consolidación de una zona de gran importancia en el esquema del turismo residencial. Los fraccionamientos creados en Avándaro no reflejaban aún ninguna consecuencia negativa para la población local, al contrario, la actividad creciente de la construcción generaba fuentes de empleo. Del mismo modo los recursos del municipio comenzaron a tener un impacto positivo derivados del cobro del impuesto predial, creciendo a su vez la demanda de servicios; es por esto que la estructura orgánica y operativa del gobierno municipal se ve modificada, ya que aumenta el grado de complejidad en el ejercicio del gobierno.

Crecimiento. La devaluación de 1982 incide en el crecimiento de Valle de Bravo en el esquema de segundas residencias y entre 1984 y 1989, sucede el "gran boom", el lugar se convierte en sitio de moda, comienza a ser considerado como un símbolo de estatus y, la dinámica se intensifica en todas las áreas. Es este probablemente el periodo de mayor 
crecimiento en el turismo residencial y se inicia una marcada tendencia a la pulverización de la tierra, esto es, que comienza un proceso en el que los grandes lotes originales del Fraccionamiento Avándaro, son subdivididos y el número de viviendas crece respecto a lo esperado.

La demanda de servicios se incrementa considerablemente, a la vez que se perciben las primeras consecuencias ambientales principalmente de deforestación. Comienza a reflejarse una problemática de vialidad, puesto que la traza urbana no admite la carga vehicular que producen tanto el crecimiento poblacional natural, como el incremento en el número de visitantes de fin de semana (población flotante), propietarios de segundas residencias. Debido a este crecimiento, se construyen nuevas vías de acceso, por lo que durante este periodo Valle de Bravo ya contaba con dos carreteras que lo contectaban con los municipios aledaños y principalmente la ciudad de Toluca, que es la conexión principal con la Ciudad de México.

En 1987 el municipio comienza a regularse en materia urbana. Con fundamento en previsiones contenidas en la Ley de Asentamientos Humanos (en ese entonces en vigor para el Estado de México), la cabecera municipal y sus áreas de influencia fueron definidas como "Centro de Población Estratégico". De este se derivó un esquema normativo denominado "Plan de Centro de Población Estratégico", orientado a lograr el ordenamiento en función de las características propias de la localidad; se define por primera vez lo relativo a los usos de suelo para el otorgamiento de licencias de construcción, imponiendo también la prohibición de derribo de árboles como medida para frenar el deterioro ambiental.

En esta etapa se destacan incrementos poblacionales altos, así como una tendencia a la especialización en los sectores secundarios y terciarios de la economía, el primero influído por la producción de electricidad, gas y agua así como la construcción; el segundo influído por el arribo de visitantes a la zona mostrando una especialización en todos sus rubros. También se destaca que durante este periodo las viviendas tuvieron un crecimiento aún mayor que el presentado por la población, denotando así la alza en la construcción de segundas residencias en el municipio.

Consolidación. Con relación a la normatividad de Valle de Bravo en materia urbana, el Plan de Ordenamiento Urbano surgido en 1987 continuó vigente para el municipio durante los años siguientes. Sin embargo, comienza a apreciarse una tendencia de "modernización" y a ser alterado el 'estilo' de construcción típico de Valle de Bravo, por esto en 1997 se pone en vigor el primer "Reglamento de Imagen Urbana" como una medida para conservar la calidad arquitectónica, y consecuentemente la imagen urbana. El 13 de marzo de 2002 entró en vigor un nuevo ordenamiento decretado por el ejecutivo estatal denominado "Código Administrativo del Estado de México", mediante el cual se derogaba la mencionada Ley de Asentamientos Humanos, aunado a esto, el gobierno 
estatal transmite facultades al municipal en lo relacionado con el desarrollo urbano y se emite el Plan Municipal de Desarrollo Urbano, vigente hasta la fecha.

La problemática de vialidad se intensifica debido, entre otras cosas, a que el municipio desde 1990 hasta 1995 tuvo una tasa de crecimiento de población superior en casi dos puntos a la registrada en todo el Estado de México. Del mismo modo para 1992 se estimó la existencia de aproximadamente dos mil casas de fin de semana, con una capacidad para alojar 16.000 personas (AMVB, 2014). Durante esta etapa se otorgaron contratos a tres empresas constructoras para la realización de la autopista Toluca - Valle de Bravo, lo que generó una gran expectativa de desarrollo. Así en agosto de 2008 se inaugura el tramo carretero que conecta a Valle de Bravo con la Ciudad de México, contribuyendo a una disminución de casi 45 minutos en el traslado.

Derivado de todo esto surge una de las problemáticas a las que se enfrenta Valle de Bravo actualmente, el de la vialidad, ya que el crecimiento natural poblacional, así como la intensificación del desarrollo urbano en las zonas residenciales han provocado que, los fines de semana y períodos vacacionales ocurran embotellamientos en el centro de la población y en los puntos de confluencia de las vías principales. Del mismo modo, se han desarrollado diversas zonas comerciales, como consecuencia de la propia demanda, originando una mayor dificultad para trasladarse de Avándaro a Valle de Bravo.

De acuerdo al modelo del Ciclo de Vida Turístico, al periodo de consolidación le sigue una etapa de estancamiento que surge cuando los niveles de capacidad de carga, es decir el número máximo de visitantes en una zona, son alcanzados o excedidos. Para el caso del turismo residencial estos niveles se pueden ejemplificar mejor a través del espacio físico, ya que los municipios tienen una zona finita de urbanización la cual no puede ser excedida una vez llegado a su límite, por lo que para identificar el escenario posible a seguir en Valle de Bravo, se analizará a continuación la demanda de uso de suelo, la tendencia de crecimiento de las segundas residencias y el crecimiento poblacional.

\section{TENDENCIA DE CRECIMIENTO DE LAS SEGUNDAS RESIDENCIAS}

En la Tabla 1, se muestra el crecimiento poblacional y de viviendas que ha mostrado VB desde 1970 al 2010. También se observan las tasas de crecimiento media anual (TCMA) poblacional y de viviendas, esto para poder mostrar cómo el crecimiento poblacional no siempre fue equilibrado con relación al crecimiento de las viviendas en el municipio. 
Tabla 1: Crecimiento poblacional y de viviendas en Valle de Bravo. 1970 - 2010

\begin{tabular}{|c|c|c|c|c|}
\hline Periodo & $\begin{array}{c}\text { Población Total } \\
\text { (PT) }\end{array}$ & $\begin{array}{c}\text { Viviendas } \\
\text { Totales (VT) } \\
\text { (Fin del periodo) }\end{array}$ & $\begin{array}{c}\text { TCMA } \\
\text { poblacional (\%) }\end{array}$ & $\begin{array}{c}\text { TCMA } \\
\text { viviendas (\%) }\end{array}$ \\
\hline $1960-1970$ & 23.779 & 4.001 & 4 & 3 \\
\hline $1970-1980$ & 36.762 & 6.301 & 4.4 & 0.9 \\
\hline $1980-1990$ & 36.135 & 6.945 & -0.2 & 3.9 \\
\hline $1990-2000$ & 57.375 & 10.235 & 4.6 & 3.7 \\
\hline $2000-2010$ & 61.599 & 14.778 & 0.67 & \\
\hline
\end{tabular}

Fuente: Elaboración propia con datos de los Censos de Población IX, X, XI, XII, XIII, INEGI y Plan Municipal de Desarrollo Urbano de Valle de Bravo 2006.

Resalta que en los periodos de 1960 - 1970 y 1970 - 1980, las tasas de crecimiento poblacional y habitacional son proporcionales, es decir que el crecimiento de la población es congruente con el aumento de viviendas; sin embargo, durante el periodo de 1980 a 1990, la población presentó una tasa de crecimiento negativo, es decir la población disminuyó con respecto al periodo anterior, pese a este crecimiento negativo la vivienda registró uno positivo por lo que ya se comenzaba a vislumbrar la presencia de las segundas residencias en la zona. Del mismo modo en el último periodo de la tabla, se vuelve a mostrar una tendencia semejante a la de 1980 - 1990, mostrando cómo el entorno urbano seguía creciendo a un ritmo diferente que el poblacional. Así entonces, mientras la población creció a una tasa promedio de $1.69 \%$ entre 1990 y 2010, las viviendas lo hicieron al $2.8 \%$ en el mismo periodo, evidenciando la expansión urbana con una dinámica superior a la demográfica lo que se explica por la preeminencia del enfoque turístico-recreativo del entorno urbano (EURE, 2014 ).

Una variable estadística fundamental para comprender la capacidad de carga que puede tener un municipio que alberga turismo residencial es la población flotante, término utilizado para estimar la cantidad de personas que viven de forma temporal en un sitio, no forman parte del conteo oficial del INEGI, pero realizan contribuciones fiscales (impuestos) y hacen uso de los servicios públicos (y en ocasiones turísticos) del municipio. Es por esto que analizar su crecimiento, su peso con relación a la población local y su tendencia futura, es muy importante para éste destino turístico.

En la Tabla 2, se muestra la cantidad de población desde 1990 hasta 2020, el estimado de segundas residencias, el cálculo de población flotante, así como el porcentaje que tiene la población flotante sobre la local. 
Tabla 2: Crecimiento poblacional y de segundas residencias en Valle de Bravo. 1990 - 2030

\begin{tabular}{|c|c|c|c|c|c|}
\hline Año & $\begin{array}{c}\text { Población } \\
\text { Total (PT) }\end{array}$ & $\begin{array}{c}\text { Segundas } \\
\text { residencias (SR) }\end{array}$ & $\begin{array}{c}\text { Promedio } \\
\text { Habitantes por } \\
\text { Vivienda Total } \\
\text { (PHVT) }\end{array}$ & $\begin{array}{c}\text { Población Flotante } \\
\text { (PF) }\end{array}$ & $\begin{array}{c}\text { PF sobre } \\
\text { PT }\end{array}$ \\
\hline 1990 & 36.135 & 2.000 & 5.2 & 10.400 & 29 \\
\hline 2000 & 57.375 & 3.364 & 5.3 & 17.834 & 31 \\
\hline 2010 & 61.599 & 5.294 & 4.2 & 22.234 & 46 \\
\hline $2020^{*}$ & 76.386 & 8.705 & 4.0 & 34.821 & 48 \\
\hline $2030 *$ & 86.404 & 11.520 & 3.6 & & 474 \\
\hline
\end{tabular}

Fuente: Elaboración propia con datos del Censo de Población y Vivienda XI, XII, XIII, INEGI. Proyecciones de población 2020-2030, CONAPO y Centro EURE S.C.

*Los datos del 2020 y 2030 corresponden a proyecciones de crecimiento.

Los datos muestran la tendencia de crecimiento que ha tenido VB con relación a las segundas residencias y por lo tanto con la población flotante. Se destaca que existe un peso importante de la población flotante sobre la local, así como una tendencia a la alza, lo cual indica que los turistas residenciales tienen una fuerte presencia en el municipio. Así mismo la proyección de crecimiento al 2030 indica que la población flotante podría alcanzar casi la mitad de la población local, dato que resulta interesante ya que esa cantidad adicional de pobladores demandarán toda clase de servicios durante su estancia, principalmente los que se relacionen con los básicos para sus viviendas.

Por otro lado estos aumentos demográficos y urbanos, han traído una serie de cambios en los usos de suelo del municipio, los cuales han condicionado de forma importante los recursos naturales de la zona, principalmente el bosque. En la Gráfica 1 se detallan los cambios ocurridos en el uso de suelo en un periodo de casi 20 años. 
Gráfica 1: Cambios en el uso de suelo en Valle de Bravo. $1994-2010$

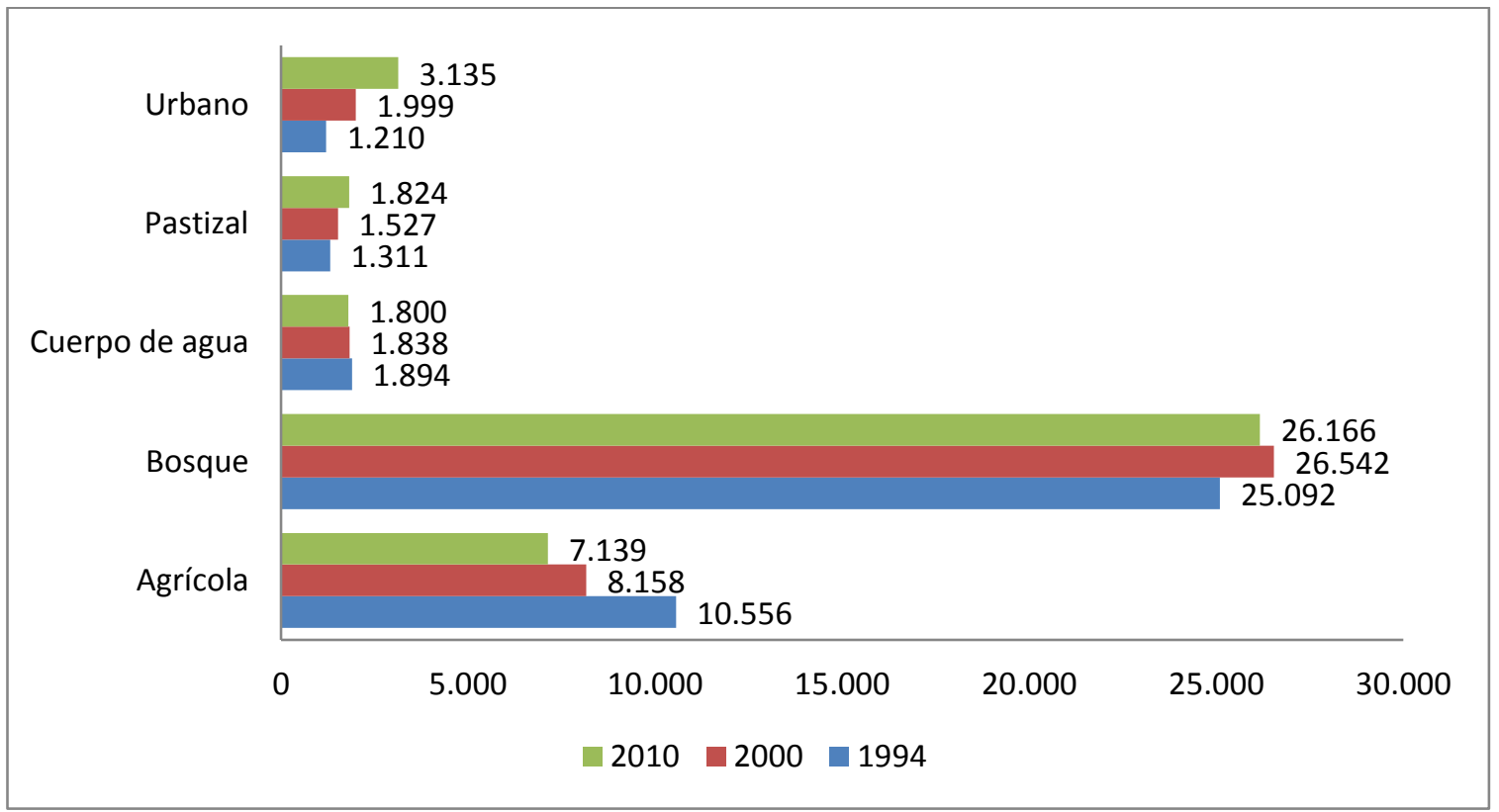

Fuente: Elaboración propia con datos de Centro EURE

De acuerdo con estos datos se observa como de 1994 al 2010 los usos de suelo en la zona, han sufrido modificaciones considerables, el suelo agrícola y los bosques muestran una disminución en cuanto a extensión se refiere, los pastizales han aumentado $y$, los cuerpos de agua por su parte, señalan una pequeña baja en cuanto a su extensión. Sin embargo lo más notable de este gráfico es el aumento en el uso del suelo urbano el cual pasó de 1.210 ha en 1994 a 3.135 en el 2010, es decir, en 16 años la urbanización creció 1.925 ha Si se profundiza en este dato, se tendrá que cada año del periodo se urbanizaron aproximadamente 120 ha.

Una vez mostrados los datos de crecimiento poblacional total y flotante, el de segundas residencias y los usos de suelo, en la Tabla 3 se muestran cuáles fueron los requerimientos de suelo en el 2010 y cuáles serán para el 2020 y 2030 . Se tiene que para el año 2000, los 57.375 habitantes se encontraban establecidos en 1.999 ha; dividiendo estos datos se obtiene que en ese año había un promedio de 28.7 habitantes por hectárea. Usando este cálculo como referencia y suponiendo que siguiera la misma tendencia de distribución de habitantes en el territorio, se obtienen los siguientes requerimientos del suelo para el 2030: 
Tabla 3: Requerimientos de suelo para Valle de Bravo. 2010 - 2030

\begin{tabular}{|c|c|c|c|c|c|c|}
\hline Año & $\begin{array}{c}\text { Población } \\
\text { Total } \\
\text { (PT) }\end{array}$ & $\begin{array}{c}\text { Población } \\
\text { Flotante } \\
\text { (PF) }\end{array}$ & $\begin{array}{c}\text { Población } \\
\text { Final } \\
\text { (PT+PF) }\end{array}$ & $\begin{array}{c}\text { Incremento } \\
\text { poblacional a partir } \\
\text { del 2000 }\end{array}$ & $\begin{array}{c}\text { Superficie } \\
\text { requerida (ha) }\end{array}$ & $\begin{array}{c}\text { Superficie } \\
\text { disponible } \\
\text { (ha) }\end{array}$ \\
\hline 2010 & 61.599 & 22.234 & 83.833 & 4.224 & 147.1 & 707.2 \\
\hline $2020^{*}$ & 76.386 & 34.821 & 111.207 & 14.787 & 515.2 & 192 \\
\hline $2030^{*}$ & 86.404 & 41.474 & 127.878 & 10.018 & 349 & -157 \\
\hline
\end{tabular}

Fuente: Elaboración propia con base en proyecciones de crecimiento poblacional 2020 - 2030. CONAPO. *Los datos de los años 2020 y 2030 corresponden a proyecciones.

El Plan Municipal de Desarrollo Urbano (2006) establece un área programada como urbanizable de 854.3 ha, por lo que para el año 2020 estaría cubierta la demanda de suelo, pero para el 2030 existiría un déficit. Ahora bien, esta área urbanizable no hace distinción entre la edificación de segundas residencias y la vivienda local, es un dato generalizado, es decir el suelo puede ocuparse indistintamente para la construcción de cualquier tipo de vivienda, de hecho un estudio realizado por el Centro EURE arroja qué de las 515.2 ha que se requieren para el 2020, el $65 \%$ del suelo sería para la construcción de viviendas tipo medio y popular, el $20 \%$ vialidades y el $15 \%$ restante equipamiento urbano; así que, si se toman en cuenta estos datos se pueden desprender dos escenarios posibles:

Primero, sí se utiliza el porcentaje de ocupación que tienen las segundas residencias con relación a la vivienda local para el 2020 (46\%), se destaca que de las hectáreas contempladas para la urbanización de acuerdo al Plan Municipal de Desarrollo Urbano, se necesitarán aproximadamente 393 hanmás a las previstas para la construcción de este tipo de viviendas, es decir, si se respeta el espacio para la demanda de casas de la gente local, para el 2020 se necesitarán 908 ha, 53.7 ha más a las previstas. Por lo que, Valle de Bravo para ese año, estaría entrando en una etapa de estancamiento, provocada por un fuerte condicionamiento de demanda del suelo, así como una posible presión para realizar construcciones en zonas boscosas o áreas naturales protegidas.

Por otro lado, se puede considerar un segundo escenario en el cual las hectáreas contempladas para la urbanización se utilicen indiscriminadamente para la edificación de viviendas locales y de segundas residencias, éstas últimas pudiendo tener un favoritismo ya que los terrenos pueden ser adquiridos por personas con mayores ingresos económicos, o que las mismas agencias inmobiliarias los adquieran para una comercialización a los turistas residenciales, provocando que los precios de éstos suban aún más haciendo difícil que la gente local pueda comprarlos y por lo tanto decidan construir sus casas en zonas de alto riesgo, alejadas de los servicios básicos, en situaciones de hacinamiento o en zonas naturales protegidas. Si bien este escenario muestra que el

\footnotetext{
${ }^{1}$ La superficie requerida se calcula dividiendo el incremento poblacional entre la población promedio por hectárea calculada.
} 
municipio podría tardar más años en alcanzar una etapa de estancamiento, representa también muchos más riesgos para la zona y la población local.

Cabe mencionar que los escenarios mostrados están relacionados con una proyección de crecimiento igual al actual, es decir que la tendencia se mantenga constante; sin embargo se puede hablar de un crecimiento mucho más lento, esto sustentado en que las mismas tasas de crecimiento poblacional disminuyan (acorde con algunas tendencias de CONAPO), por lo que la expansión urbana puede desacelerarse y provocar que Valle de Bravo cuente con más años para lograr su consolidación completa. También es importante señalar que amenazas externas a la misma población y turistas residenciales, pueden frenar el crecimiento, tal es el caso de la inseguridad que ha vivido la zona (y el país en general) en los últimos años.

\section{CONCLUSIONES}

De acuerdo a lo planteado anteriormente, se puede concluir que Valle de Bravo se encuentra en una etapa de consolidación de acuerdo al modelo CVT; según los cálculos y estimaciones presentadas, para el 2030, dependiendo del ritmo de crecimiento, podría estar entrando en una etapa de estancamiento, es decir sus niveles de capacidad de carga estarían llegando a su punto máximo, esto debido principalmente a que el suelo urbanizable se verá fuertemente condicionado por el crecimiento poblacional.

Aunado a esto, se presenta la problemática de que ante la falta paulatina de suelo urbanizable, el bosque se vea fuertemente propenso a seguir siendo utilizado para la construcción de viviendas; por lo que es necesario aplicar las leyes de protección ambiental y evitar que uno de los valores paisajísticos más importantes de Valle de Bravo se pierda. Así mismo al estar alcanzando límites de capacidad de carga se hará evidente una disminución en el abasto de recursos, principalmente en el agrícola y en los cuerpos de agua, trayendo problemas no sólo para los turistas residenciales, sino para la población local.

Se mostró en el análisis que la cantidad de suelo urbanizable no será suficiente dentro de los próximos años para la demanda de viviendas, lo que puede llevar a pensar que las autoridades comenzarán a considerar el crecimiento urbano en zonas aledañas al municipio, tal es el caso de Colorines, localidad importante en términos de expansión urbana y captación poblacional. Esta localidad ya se encuentra incluida en el Plan Municipal de Desarrollo Urbano (2006) buscando su ordenamiento territorial definiendo sus zonas urbanizables y no urbanizables, así como diversas estrategias de planificación.

Por otro lado se observa qué, si el municipio alcanzara su etapa de estancamiento, en los próximos años, la siguiente etapa sería el declive, la cual, como señala el modelo CVT, presenta posibles escenarios de los cuales el más deseable para el municipio sería el de rejuvenecimiento, es decir, la búsqueda de nuevas formas de turismo que sigan 
manteniendo la zona vigente y así, hipotéticamente, iniciar un nuevo ciclo. Una de las propuestas más interesantes surge del mismo gobierno estatal y municipal, donde se está buscando ya la promoción de turismo ecológico, de aventura y de salud.

Resumiendo, las autoridades de Valle de Bravo necesitan implementar correctamente toda su normatividad vigente para evitar el declive total de la zona, requiere tomar en cuenta seriamente al turismo residencial y a la población flotante que de él emerge para realizar proyecciones inmediatas más precisas relacionadas con su crecimiento y ocupación espacial, pues como se mostró anteriormente, las segundas residencias han condicionado en gran medida el desarrollo municipal. Así mismo se deben de planear acciones que ayuden a diversificar al turismo para que al momento de alcanzar la etapa de estancamiento pueda conducirse a un rejuvenecimiento y la zona siga generando beneficios positivos para todos sus habitantes.

\section{REFERENCIAS BIBLIOGRÁFICAS}

Alarcón, P., González, E. y Pérez, J. (2010). Residential tourism in the south of Spain: an approach towards consumption. Revista Encontros Científicos - Tourism \& Management Studies, 6, 36-48.

Aledo, A. (septiembre 2002). Turismo Inmobiliario y la fagotización de la naturaleza. Artículo presentado en el Congreso Recreaciones Medioambientales, Políticas de Desarrollo y Turismo, Barcelona, España.

Aledo, A. (2008). De la tierra al suelo: la transformación del paisaje y el nuevo turismo residencial. Arbor, 184(729), 99-113.

Aledo, A., García, H., y Ortíz, G. (2010). Análisis de mapas causales de impactos del turismo residencial. EMPIRIA, revista metodológica de ciencias sociales, 20, 61-86.

Aledo, A., Mazón, T., Mantecón, A. y Lagunes, D. C. (2007). La insostenibilidad del turismo residencial. En D. Lagunes (Coord.), Antropología y Turismo: claves culturales y disciplinares (pp. 185-208). México D.F: Plaza y Valdés.

Almeida, F. y López, D. (2003). La costa del sol oriental: un conflicto territorial entre el turismo, la agricultura y la organización metropolitana Baetica. Estudios de arte, geografía e historia, 25, 67-80.

AMVB. (2014). Archivo Municipal de Valle de Bravo.

Benson, M. y O'Reilly, K. (2009). Migration and the search for a better way of life: a critical exploration of lifestyle migration. The sociological review, 57, 608-625.

Casado, M., Kaiser, C. y Warnes, A. (2004). Northen European retired residents in nine southern European areas: characteristics motivations an adjustment. Ageing and society, 24 (3), 353-381.

Coles, T., Duval, D. y M., H. (2005). Sobre el turismo y la movilidad en tiempos de movimiento y conjetura posdiciplinaria. Política Y Sociedad, 42(2), 181-198. 
Díaz, F. (2004). Turismo, urbanización y cambio social. Revista Argentina de Sociología, 2, 115-127.

Díaz, F. y Lourés, M. (julio 2006). Housing, tourism and the real estate sector: the Spanish mediterranea coast. Trabajo presentado en ENHR Conference: Housing in an expanding Europe, Ljubljana, Slovenia.

Fernández, S. y Barrado, D. (2011). El desarrollo turístico inmobiliario de la España mediterránea Insular frente a sus referentes internacionales (Florida y la Costa Azul): un análisis comparado. Cuadernos de Turismo, 27, 373-402.

García, A., Artal, A. y Ramos, J. (2002). El turismo del mar menor: predominio de la segunda residencia. Cuadernos de turismo, 9, 33-43.

García, B., Reinares, E. y Armelini, G. (2013). Ciclo de vida de los destinos turísticos y estrategias de comunicación: los casos de España y Chile. Revista Internacional de Investigación en Comunicación aDResearch ESIC, 7(7), 76-93.

Gili, M. (2003). Las viviendas de segunda residencia ¿Ocio o negocio? Scripta Nova. Revista electrónica de geografía y ciencias sociales, 7, 52.

Gobbels, C. (1954). Geografía de Valle de Bravo. Estado de México.

Guilding, C., Warnken, J., Ardill, A. y Fredline, L. (2005 ). An agency theory perspective on the owner/ manager relationship in tourism - based condominiums. Tourism Management, 26, 409-420.

Hall, C. M. y Müller, D. K. (2004). Tourism, mobility, and second homes: between elite landscape and common ground. Clevedon: Channel View Publications.

Harrison, D. (1995). Development of Tourism in Swaziland. Annals of Tourism Research, 22(1), 135-156.

Hiernaux, D. (2004). El turismo de segundas residencias en México. México: Universidad Autónoma Metropolitana Iztapalapa - Secretaría de Turismo.

Huete, R. y Mantecón, A. (2010). Los límites entre el turismo y la migración residencial. Una tipología. Papers: revista de sociologia, 95(3), 781-801.

Huete, R., Mantecón, A. y Mazón, T. (2008). ¿De qué hablamos cuando hablamos de turismo residencial?. Cuadernos de turismo, 22, 101-121.

Huete, R., Mantecón, A. y Mazón, T. (2008). Tendencias del turismo residencial: el caso del Mediterráneo Español. Periplo Sustentable, 14, 65-87.

Iracheta, A., Iracheta, J. y Sánchez, M. a. (noviembre 2014). El agua es primero: crisis y oportunidad en Valle de Bravo, México. Artículo presentado en el II Congreso Iberoamericano de Suelo Urbano, La Habana, Cuba.

López, D. (1995). La inmigración en España a fines del siglo XX. Los que vienen a trabajar y los que vienen a descansar. REIS, 71, 225-245.

Lundtorp, S. y Wanhill, S. (2001). The Resort Lifecycle Theory. Generating Processes and Estimation. Annals of Tourism Research, 28(4), 947-964.

Mantecón, A. y Huete, R. (2011). Sociological Insights on residential tourism: host society attitudes in a mature destination. European journal of tourism research, 4(2), 109-122.

Mazón, T. (2006). El turismo litoral mediterráneo: ¿políticas turística o desarrollo inmobiliario?. Barcelona: Icaria. 
Mazón, T. y Aledo, A. (2005). El dilema del turismo residencial: ¿turismo o desarrollo inmobiliario?. En T. Mazón y A. Aledo (Eds.), Turismo residencial y cambio social: nuevas perspectivas teóricas y empíricas (pp. 13-30). Universidad de Alicante.

Mazón, T., Huete, R. y Mantecón, A. (2009). La dependencia del turismo y la percepción de la comunidad de acogida. Apuntes sobre la teoría del intercambio social. Encontros científicos-tourism \& management studies, 5, 25-36.

Módenes, J. A. (junio 2006). Movilidad Espacial: Uso Temporal del Territorio y Poblaciones Vinculadas. Artículo presentado en el X Congreso de la Población Española: "Migraciones, movilidad y territorio", Pamplona, España.

Müller, D. (2002). German second homeowners in Sweden. Some remarks on the tourism-migrations-nexus. Revue européene des migrations internationals, 18(1), 67-86.

O'Really, K. y Benson, M. (2009). Lifestyle migrations: escaping to the good life. En M. Benson y K. O’Really (Eds.), Lifestyle migrations: Expectations, Aspirations and Experiences (pp. 1-13). Farnham: Ashgate.

PMDU (2006). Plan Municipal de Desarrollo Urbano. Valle de Bravo: Gobierno del Estado de México.

PMDVB (2013). Plan Municipal de Desarrollo de Valle de Bravo. Valle de Bravo: Gobierno del Estado de México.

Rodríguez, V. (2004). Turismo residencial y migración de jubilados. Colección Mediterráneo. Las nuevas formas del turismo, 5, 233-253.

Salvá, P. (2011). El turismo residencial ¿una manifestación de nuevos turismo y nuevos comportamientos turísticos del siglo XXI?. Cuadernos de turismo, 27, 823-836.

Sanz, S. (2008). Imagen global e intenciones futuras del comportamiento del turista de segunda residencia. Revista europea de dirección y economía de la empresa, 17(4), 95-114.

Serra, C. A. (2011). Marketing turístico. Madrid: Pirámide.

Torres, E. (2003). El turismo residencial y sus efectos en los destinos turísticos. Estudios turísticos, 155-156, 45-70.

UAM, CESTUR (2004). El Turismo de Segundas Residencias en México. México. Universidad Autónoma Metropolitana y Centro de Estudios Turísticos

Varela, B., López, A. y Martínez, A. (2003). Primeras aproximaciones al estudio estadístico del alojamiento privado con fines turísticos desde una perspectiva de oferta. Estudios turísticos, 155, 87-109.

Vera, J. F., López Palomeque, F., Marchena, M. y Anton, S. (1997). Análisis territorial del turismo. Barcelona: Ariel.

Williams, A. M. y Hall, C. M. (2000). Tourism and migration: New relationships between production and consumption. Tourism Geographies: An International Journal of Tourism Space, Place and Environment, 2(1), 5-27. doi: $10.1080 / 146166800363420$ 